\title{
A green light for plants
}

\author{
MICHAE JASIŃSKI \\ ${ }^{1}$ Department of Biochemistry and Biotechnology, Poznań University of Life Sciences, Poland \\ ${ }^{2}$ Institute of Bioorganic Chemistry, Polish Academy of Sciences, Poznań, Poland \\ e-mail: jasinski@ibch.poznan.pl
}

Global demand for food, feed, and fuel displays a permanent increase. Recently, it has become one of the most pressing problems which humanity faces. An example might be the worldwide consumption of grains (food and biofuel) which has already outpaced production, resulting in the lowest level of the world grain stock since 1960 (Brown, 2008). The projected increase in the global population, along with the climate change, has only exacerbated these problems.

To satisfy the growing demand for adequate nutrition we could simply start with proper distribution of what we already have in the developed countries. However, due to complex political and economical interplays of interests, this is still far from being possible. It looks as though under the shrinking resources of cultivable area and irrigation, the only reasonable solution for now is to improve the productivity. Considering this in a broad field of biology, plant science or plant biotechnology has a central role in delivering new agricultural varieties which will help overcome food security and sustainability problems.

Proper strategies, based not only on traditional cultivation procedures supported with machinery and fertilizers, but also on the introduction of new traits, as well as the development of a new breeding methodology with DNA markers are the ways to improve the green production. This is illustrated, for instance, by the history of an increase in corn yield in the United States from 1.6 tones/ha at the beginning of $20^{\text {th }}$ century up to 9.5 tones/ha nowadays (Edgerton, 2009).

Several plant genome sequences are fully deciphered (e.g., rice, soybean, poplar, grape) while others, like cotton, Medicago, or tomato are in the pipeline. We are now able to explore large gene groups in order to look for shared or specific structural or functional features. The next-generation sequencing technologies help us in understanding the role of epigenetics that regulates gene expression in various cultivars. Such sequencing finds its way into plant genetics and promises substantial improvements in crop yields over the coming years. An example of it might be the construction and use of a haplotype map for genome-wide association studies (GWAS). GWAS helps to determine the genetic basis of crop traits and is a milestone in identifying superior genetic variants to be used in breeding (Lam H.-M. et al., 2010). Beyond genomes, platforms to study expression patterns at the RNA level (e.g., micro-arrays) as well as diverse integrated approaches, including advanced proteomic techniques combined with bioinformatics, metabolomics and molecular cell biology, allow for the better understanding of the functional roles of genes. This knowledge will significantly improve green technologies. Progress also has been made with the application of microRNA in agriculture. We can now use microRNAs to control gene expression and double-stranded RNAs for pest protection (Gordon and Waterhouse, 2007).

Our view on genetically engineered plants is changing; it is a slow but required process. It becomes apparent that benefits of transgenic crops can outweigh their potential environmental risks. Molecular breeding based on solid knowledge is not far from natural breeding and allows for much faster development of new varieties. Such varieties, e.g. modified to resists pathogenic infections, offer reduction in chemical warfare that is commonly employed nowadays, thus producing a positive impact on the environment. In addition to the biotic stress control, one of the most important and probably soon commercialized new agricultural trait which will boost crop production is improved drought tolerance. Water deficit is a major threat even in the most productive regions. Fresh water is a luxury and we should not forget that $70 \%$ of globally available fresh water is used to sus- 
tain green production. Obtaining new, drought-tolerant varieties will allow their cultivation in dried areas and decrease water requirement.

Drought stress response is associated with changes in gene expression profile. Many of stress-induced genes are known or presumed to play key roles in drought tolerance. An emerging strategy is to identify so-called master regulatory genes involved in water usage in plant. Such genes, encoding, for instance, transcription factors, play a predominant role in the control of wide expression networks and are promising for the modification of complex traits. They offer a new factor based on nextgeneration biotechnologies. Identification of the crucial transcription factors is one task, while understanding the signaling cascade remains difficult but an equally promising issue. Recently, expression of certain genes encoding transcription factors associated with drought stress tolerance has been shown to be regulated posttranscriptionaly via miRNA (Li et al., 2008). However, despite the importance of drought tolerance, no small RNAs regulating drought stress responses have been found so far.

Plant hormone abscisic acid (ABA) is a key signaling intermediate controlling expression of several stress-induced genes. It coordinates responses to abiotic stresses such as drought, extreme temperature and high salinity. Recent discoveries of how this plant hormone transmits messages together with identification of its receptor (PYR1) (Santiago et al., 2009, Miyazono et al., 2009) pave the way towards the new concepts of controlling drought tolerance. In addition to the known ABA signaling pathway, recently the molecular basis of ABA transport have been discovered. It has been shown that members of the ABCG subfamily of ATP-binding cassette $(\mathrm{ABC})$ proteins are responsible for $\mathrm{ABA}$ transport. Interestingly, members of this subfamily participate in the export of ABA through the plasma membrane, being involved in the signaling pathway within the extracellular environment of the guard cells, and in the import of ABA through a plasma membrane, into guard cells thus integrating ABA-dependent signaling and transport processes (Kuromori et al., 2010; Kang et al., 2010). Manipulation of ABA transport may additionally result in the development of plants that quickly respond to environmental stresses.

A major challenge in the sustainable agriculture also comprises the production of high quality plant material without any negative impact on the environment. Nitrogen $(\mathrm{N})$ and phosphorus $(\mathrm{P})$ play significant roles in intensive agriculture, the same time the application of chemical fertilizers is a major cause of ecosystem pollution. Moreover, production of such chemicals is highly energy-consuming. In spite of this, particular attention is being paid toward the usage of legumes and symbiotic $\mathrm{N}_{2}$ fixation to improve soil $\mathrm{N}$ and $\mathrm{P}$ fertility. Legume plants can establish symbiosis with nitrogen fixing bacteria, hence the independence from nitrogen fertilizers. Furthermore, they efficiently establish mycorrhizal associations, which allow the plant to efficiently use phosphate even if tightly absorbed onto the surface of solid matter in the soil. Ongoing fundamental research, helping to understand the role of individual genes in improving $\mathrm{N}$ and $\mathrm{P}$ use and assessing the factors limiting rhizobial and mycorrhizal interactions with plants, can help to engineer nitrogen-fixing crops. This is definitely not easy but an exciting challenge. Despite the fact that our understanding of nodule organogenesis in legumes is advancing, it is unlikely that a single engineering event will allow us to obtain new nitrogen-fixing varieties. However, at least a part of the signaling pathway necessary for the establishment of successful colonization is present in cereals and probably we need not to transfer every genetic component defined in legumes (Charpentier and Oldroyd, 2010). From the evolutionary point of view, formation of nodules, as a new structure in legumes, appeared by adaptation of the pre-existing developmental processes (e.g., lateral root formation) and signaling molecules (hormones) related to them. Later research has underlined the role of plant hormones like auxin and cytokinins in the nodulation specific developmental processes. Understanding what defines the specific response of legumes for these hormones remains the major challenge in the upcoming years.

As always when new information is being gathered, there is a possibility to use it in the field of applied plant research. However, the biggest challenge is to demonstrate the potential public benefits of green biotechnology. This tackles not only the assurance of sustainability in the management of natural resources but, more importantly, the need of scientific community to educate consumers to comprehend and be more comfortable with new solutions based on appropriate scientific methods. 


\section{References}

Brown L.R. (2008) World facing huge new challenge on food front - Business-as-usual not a viable option. Earth Policy Institute (http://www.earthpolicy.org/Updates/2008/ Update72.htm)

Charpentier M., Oldroyd G. (2010) How close are we to nitrogen-fixing cereals? Curr. Opin. Plant Biol.13(5): 556-564.

Edgerton M. (2009) Increasing crop productivity to meet global needs for feed. Food Fuel Plant Physiol. 149: 7-13.

Gordon K.H., Waterhouse P.M. (2007) RNAi for insect-proof plants. Nat. Biotechnol. 25(11): 1231-1232.

Kang J., Hwang J.U., Lee M., Kim Y.Y., Assmann S.M., Martinoia E., Lee Y. (2010) PDR-type ABC transporter mediates cellular uptake of the phytohormone abscisic acid. Proc. Natl. Acad. Sci. USA 107(5): 2355-2360.

Kuromori T., Miyaji T., Yabuuchi H., Shimizu H., Sugimoto E., Kamiya A., Moriyama Y., Shinozaki K. (2010) ABC transporter AtABCG25 is involved in abscisic acid transport and responses. Proc. Natl. Acad. Sci. USA 107(5): 2361-2366.

Lam H.M., Xu X., Liu X., Chen W., Yang G., Wong F.L., Li M.W., He W., Qin N., Wang B., Li J., Jian M., Wang J.,
Shao G., Wang J., Sun S.S., Zhang G. (2010) Resequencing of 31 wild and cultivated soybean genomes identifies patterns of genetic diversity and selection. Nat. Genet. 42(12): 1053-1059.

Li W.X., Oono Y., Zhu J., He X.J., Wu J.M., Iida K., Lu X.Y., Cui X., Jin H., Zhu J.K. (2008) The Arabidopsis NFYA5 transcription factor is regulated transcriptionally and posttranscriptionally to promote drought resistance. Plant Cell. 20(8): 2238-2251.

Miyazono K., Miyakawa T., Sawano Y., Kubota K., Kang H.J., Asano A., Miyauchi Y., Takahashi M., Zhi Y., Fujita Y., Yoshida T., Kodaira K.S., Yamaguchi-Shinozaki K., Tanokura M. (2009) Structural basis of abscisic acid signalling. Nature 462(7273): 609-614.

Santiago J., Dupeux F., Round A., Antoni R., Park S.Y., Jamin M., Cutler S.R., Rodriguez P.L., Márquez J.A. (2009) The abscisic acid receptor PYR1 in complex with abscisic acid. Nature 462(7273): 665-668. 